\section{Concerning the Report on Chloroxylenol-Containing Antiseptic: Queries and Response}

\section{To the Editor:}

This letter is in reference to an article appearing in the April 1986 issue of Infection Control. The title of the article is "Brief Report: The antiseptic efficacy of chloroxylenol-containing vs. chlorhexidine gluconate-containing surgical scrub preparations." The authors are Soulsby, Barnett, and Maddox. ${ }^{1}$

1 have spent many years reviewing studies and designing protocols for skin microbiology and, in fact, developed the protocol for the Glove Juice Test which has been published in the Federal Register. ${ }^{2}$ This protocol was reviewed by various people in government, academia, and industry before it was finalized by the FDA, OTC Antimicrobial I Panel. The statistics were worked out in the Division of Biometrics at FDA. The authors refer to Dr. Peterson having developed the test. This is simply not true. Furthermore the selection of 3- and 6-hour sampling was an alteration suggested and permitted by FDA, not originated by Dr. Peterson. He simply published the results of a test he performed commercially.

I also represent a company who utilizes chloroxylenol (PCMX) in their products and are attempting to develop a reliable body of data about this antimicrobial ingredient. In this vein, there are numerous errors, miscalculations and misstatements in this article which need to be clarified.

The authors, Soulsby et al, state that chloroxylenol (PCMX) is the same chemical family as chlorhexidine gluconate. Chloroxylenol is a chlorine-substituted xylenol (phenol derivative) and chlorhexidine is a biguanide-there is no similarity.

The authors have decided that dilution is an adequate means of neutralization. I think the argument has been adequately made that chemical neutralizers are preferred. ${ }^{2}$ For example, chlorhexidine gluconate is notoriously difficult to neutralize, and some conflicting data ${ }^{3}$ may be explained in some part by incomplete neutralization. Did the authors test the adequacy of this procedure prior to use?

The authors selected counts of greater than $1 \times 10^{5}$ as the baseline count. There is bound to be significant variation in the baseline values since frequently counts at $10^{6}$ are recorded. The range of counts is then from $1 \times 10^{5}$ to more than $1 \times 10^{6}$. The original intent of the protocol was to keep the baseline count in a narrow range.

The chloroxylenol was dispensed uniformly in a prepackaged sponge and the chlorhexidine gluconate was used ad libitum with a dry sponge. What is the consistency of dosages?

I have some problem in accepting that this paper was ever reviewed by your editors. The most novice person in disinfection and sterilization should be able to detect miscalculations. If the $\log$ reductions are examined the following is observed. (I am giving only one example.)

5.6324 (baseline-average)

4.8846 (day 1 -average)

$0.7478 \mathrm{log}$ reduction

The authors report a $\log$ reduction of 1.9145 and a percent reduction of 82.1234. Unless I have been wrong for twenty years, 90 percent reduction is one log. I have a feeling the authors added an extra log conversion in their procedure. I have chosen not to recalculate their entire study. It would be interesting to see the raw data. It is fairly obvious that the reductions are similar for both products. This is most interesting since they did not use accepted chemical neutralizers, and chlorhexidine should be more difficult to neutralize than chloroxylenol.

The paper by Rotter ${ }^{3}$ shows clearly that other investigators have obtained reductions similar to the actual ones obtained by these authors, if their math were done correctly.

I am further surprised to have to correct the spelling of chloroxylenol.

The authors also imply that FDA utilizes standard products for comparison. FDA does not use comparative effectiveness or make judgments about it. Instead, products are judged on risk/benefit using objective testing.

The statements in the article regarding toxicity are sometimes out- 
of-date and based on fairly superficial summaries in the sources reviewed. Large volumes of toxicity data are on file at FDA.

The effect of substantivity after washing with both these ingredients seems to confirm the reports already in the literature. These authors have chosen only certain elements out of the original Glove Juice Protocol and based their conclusions on miscalculation. I certainly think some changes in the Glove Juice Protocol are needed, but haphazard ones do a disservice to the products and to good science.

\section{REFERENCES}

1. Soulsby ME, Barnett JB, Maddox S: Brief report: The antiseptic efficacy of chloroxylenol-containing vs. chlorhexidine gluconate-containing surgical scrub preparations. Infect Control 1986; 7(4): 223-226.

2. Federal Register, September 13, 1974; January 6, 1978.

3. Rotter ML: Povidone-iodine and chlorhexidine gluconate-containing detergents for disinfection of hands, J Hosp Infect I981; 2:273-280.

\section{Mary K. Bruch \\ Vice-President-Quality Assurance Dexide, Inc. \\ Fort Worth, Texas}

\section{To the Editor:}

I have recently read the article by Soulsby et al, ${ }^{1}$ wherein they compare a chloroxylenol-containing surgical scrub to Hibiclens. Since I am a supporter of the use of chloroxylenol in the proper circumstances, I find such work distressing. Allow me to address some of the points that I feel are incorrect.

The first point is the spelling of the material known chemically as 3,5 dimethyl, p-chloro-xylenol. This is also known as chloroxylenol, not chlorxylenol.

Another point is that while chloroxylenol is indeed a phenolic, chlorhexidine gluconate is a salt of a biguanide cation. They are not in the same chemical family.

There is no indication of the amount of either preparation that was employed in the test scrubs. It is well known that sponge material is capable of binding ingredients that are placed in contact with the sponge. This can include the chlorhexidine.

\begin{tabular}{|cccc|}
\hline \multicolumn{4}{c|}{ Log Reduction } \\
\hline & Hours & Anti Sept & Hibiclens \\
\hline Day 1 & 0 & .7478 & .6429 \\
& 3 & .4436 & .4789 \\
& 6 & .0995 & .1793 \\
Day 2 & 0 & .8920 & .7353 \\
& 3 & .4967 & .2607 \\
& 6 & .1062 (incr.) & .0424 \\
Day 3 & 0 & 1.0953 & 1.0676 \\
& 3 & $1.4405 ?$ & .6664 \\
& 6 & .1957 & .2953 \\
\hline
\end{tabular}

The most distressing issue is their results. An $82 \%$ reduction is not a 1.9 $\log$ reduction; after all, a $90 \%$ reduction is only a $1.0 \mathrm{log}$ reduction (eg, 100 $-10=90$, or $\log 100-\log 10=1$ ). The only way that they can obtain their data in Table 2 is to take the log of 82 , which indeed is 1.91 . However, $82 \%$ is not 82 but 0.82 , a difference of a factor of 100 .

Using their data in Table 1 to construct the proper table leads to the values shown above.

After having spent the past few years dealing with the activity of various antimicrobial preparations, I would consider both of these products to be inadequate for use as a surgical scrub, or the test is suspect. The data supplied by Dexide, Inc. on their chloroxylenol preparation shows it to be substantially more efficacious than either product showed in this test. Also, there are a number of independent studies on Hibiclens that would make this study suspect.

I would hope that the authors would submit a detailed (including raw data) correction so that this study can be properly evaluated.

\section{REFERENCES}

1. Soulsby ME, Barnett JB, Maddox S: Brief report: The antiseptic efficacy of chloroxylenol-containing vs. chlorhexidine gluconate-containing surgical scrub preparations. Infect Control 1986 ; $7(4): 223-226$.

\section{M.E. Garabedian, PhD} Arlington, Texas

\section{To the Editor:}

I was disappointed to read the report by Soulsby et al published in the April issue. ${ }^{1}$ I was somewhat confused in my attempt to decipher the data presented in this report and question some of the conclusions based on these data. Of principal confusion were the data transformation steps performed to obtain the "log reduction" and "percent reduction" values given in Table 2 as derived from the actual log bacterial count data in Table 1.

The high initial dilution of the hand samples (ie, 1:10,000) as stated in the Methods section of the paper dictates a minimum log recovery of 4.00 per hand. From this fact and the baseline values given in Table 1 , one can calculate that $\log$ reduction values of greater than 1.6 and 1.7 for Anti Sept and Hibiclens, respectively, are impossible. Yet, $\log$ reduction values of 1.9 are reported in Table 2.

Also, the authors apparently derived the percent reduction values in Table 2 by taking the antilog of the corresponding log reduction values. This is not correct. Actual numbers for this parameter should be close to $99 \%$ for all of the reductions reported. Contrary to the authors' statement in the Results section, there is no significant difference between any of these reduction values.

Certain statements in the report raise several other questions that should have been corrected or clarified prior to publication. These relate primarily to test methodology and data analysis which leave the reader wondering how specific conclusions were drawn. For example, $1 \mathrm{~mL}$ from a $50 \mathrm{~mL}$ sample into $299 \mathrm{~mL}$ does not 